\title{
Bi-level optimal toll design problem solved by the inverse Stackelberg games approach
}

\author{
K. Staňková ${ }^{1}$, G. J. Olsder ${ }^{1} \&$ M. Bliemer ${ }^{2}$ \\ ${ }^{1}$ Delft Institute of Applied Mathematics, Faculty of Electrical \\ Engineering, Mathematics and Computer Science, \\ Delft University of Technology, the Netherlands \\ ${ }^{2}$ Department of Transport and Planning, Faculty of Civil Engineering and \\ Geosciences, Delft University of Technology, the Netherlands
}

\begin{abstract}
We consider the special type of Stackelberg games known as inverse (or reverse) Stackelberg games and their application to the problem of bi-level optimal toll design in road traffic systems. On a given strongly connected network we assume a noncooperative game with two levels of players: the road authority as a leader and travelers as followers. We consider the road authority with two possible objectives: It either tries to maximize the total toll revenue or to minimize the total travel time of the network by setting tolls on tollable links, while the travelers minimize their travel costs by choosing their travel behavior.

In the analysis of traffic systems, link-travel times are modeled as link performance functions, relating travel time to the volume of traffic on the link. These functions are typically smooth, nonlinear, positive, and strictly increasing with link flow. We define link tolls as functions of one or more link flows of the network. Starting from small networks with several origin-destination pairs we analytically find the optimal toll functions for the road authority with the lower level defined by the deterministic user-equilibrium model.

The main contributions of this paper are the application of the new field of inverse Stackelberg games in traffic problems and finding the solution to these problems analytically. To define the toll as a function of the link flows seems to be very practical and can help to solve, for example, the congestion problems in densely populated areas.
\end{abstract}

Keywords: Stackelberg games, inverse Stackelberg games, bi-level optimal toll design problem, deterministic (Wardrop) user equilibrium. 


\section{Introduction}

Inverse (or reverse) Stackelberg games have become the subject of the recent game theory research, as a special type, or as an extension, of the Stackelberg games $[1,2]$. In the basic concept we have two players: a leader and a follower. They both try to maximize their respective objective functions, which are generally not the same; their goals are different. The leader announces his strategy as a mapping from the follower's decision space into his own decision space. The follower chooses his strategy knowing the leader's announcement of the leader's strategy in order to maximize the follower's profit. The leader, provided he is rational, can calculate in advance, what strategy will the follower choose, and with this information he can announce the strategy most profitable for him.

Although at this moment only little theory about inverse Stackelberg games is available (with for instance theorems about existence, uniqueness), and the theory is still in its infancy by discovering phenomena by means of examples, there seem to be many problems in various fields, which can be treated within its frame.

One of the fields is the bi-level optimal toll design problem (road pricing) [3]. We define inverse Stackelberg game on given road networks and find the optimal strategy for the road authority as the leader, while travellers as followers maximize the utility of their trip. We consider two possible objective functions of the road authority and discuss the properties of the solution.

The paper is composed as follows: In Section 2 we introduce the inverse Stackelberg game theory. In Section 3 we explain the bi-level optimal toll design problem. In Section 4 we present several case studies and in Section 5 we summarize the results obtained and propose some future research.

\section{Inverse Stackelberg games}

Let us consider two players, called a leader and a follower respectively, each having his own objective function, $\mathcal{J}_{\mathrm{L}}\left(u_{\mathrm{L}}, u_{\mathrm{F}}\right), \quad \mathcal{J}_{\mathrm{F}}\left(u_{\mathrm{L}}, u_{\mathrm{F}}\right)$, respectively, where $u_{\mathrm{L}}, u_{\mathrm{F}} \in \mathbb{R}$ are the leader's and the follower's decision variables. Each player chooses his own decision variable in such a way as to maximize his own objective function. The problem as stated so far could be solved for instance according to Nash or Pareto equilibrium concept [1], but also to the inverse Stackelberg equilibrium concept [4]. In the latter, one player, the leader, announces (and commits himself to) a function $\gamma_{\mathrm{L}}(\cdot)$ which maps $u_{\mathrm{F}}$ into $u_{\mathrm{L}}$. Given the function $\gamma_{\mathrm{L}}(\cdot)$, the follower will make his choice $u_{\mathrm{F}}$ according to

$$
u_{\mathrm{F}}^{*}=\arg \max _{u_{\mathrm{F}}} \mathcal{J}_{\mathrm{F}}\left(\gamma_{L}\left(u_{\mathrm{F}}\right), u_{\mathrm{F}}\right)
$$

Optimized quantities will be denoted by *. The leader, before announcing his $\gamma_{\mathrm{L}}(\cdot)$, will realize how the follower will play, and he should exploit this knowledge in order to choose the best possible $\gamma$-function, such that his own objective function 
$\mathcal{J}_{\mathrm{L}}$ becomes as high as possible. Symbolically we may write

$$
\gamma_{\mathrm{L}}^{*}(\cdot)=\arg \max _{\gamma_{\mathrm{L}}(\cdot)} \mathcal{J}_{\mathrm{L}}\left(\gamma_{L}\left(u_{\mathrm{F}}^{*}\left(\gamma_{\mathrm{L}}(\cdot)\right)\right), u_{\mathrm{F}}^{*}\left(\gamma_{\mathrm{L}}(\cdot)\right)\right) .
$$

In this way one enters the field of composed functions, which is known to be a notoriously complex area. From here onward it turns out to be very difficult to proceed in an analytic way. However, there is a trick that often works as shown in the following example.

\section{Example 2.1 Suppose}

$$
\mathcal{J}_{\mathrm{L}}\left(u_{\mathrm{L}}, u_{\mathrm{F}}\right)=50 u_{F}-125-5 u_{F}^{2}-u_{L}^{2}, \mathcal{J}_{\mathrm{F}}\left(u_{\mathrm{L}}, u_{\mathrm{F}}\right)=u_{\mathrm{L}} u_{\mathrm{F}}-u_{\mathrm{L}}^{2}-u_{\mathrm{F}}^{2} .
$$

If both the follower and the leader would maximize $\mathcal{J}_{\mathrm{L}}\left(u_{\mathrm{L}}, u_{\mathrm{F}}\right)$, the follower totally disregarding his own objective function, the leader would obtain his team maximum, i.e. $\mathcal{J}_{\mathrm{L}}(0,5)=0$. Now the leader should choose the curve $u_{\mathrm{L}}=\gamma_{\mathrm{L}}\left(u_{\mathrm{F}}\right)$ in such a way that the team maximum $\left(u_{\mathrm{L}}=0, u_{\mathrm{F}}=5\right)$ lies on this curve and moreover that this curve does not have other points in common with the set $\mathcal{J}_{\mathrm{F}}\left(u_{\mathrm{L}}, u_{\mathrm{F}}\right)=u_{\mathrm{L}} u_{\mathrm{F}}-u_{\mathrm{L}}^{2}-u_{\mathrm{F}}^{2} \leq \mathcal{J}_{\mathrm{F}}(0,5)=-25$. An example of such a curve is $u_{\mathrm{L}}=2 u_{\mathrm{F}}-10$. With this choice of the leader, the best for the follower to do is to maximize

$$
\mathcal{J}_{\mathrm{F}}\left(2 u_{\mathrm{F}}-10, u_{\mathrm{F}}\right)=-3 u_{F}^{2}+30 u_{F}-100,
$$

which leads to $u_{\mathrm{F}}=5$. Hence $u_{\mathrm{L}}=0$, and the leader obtained his team maximum in spite of the fact that the follower maximized his own objective function (however, subject to the constraint $\left.u_{\mathrm{L}}=\gamma_{\mathrm{L}}\left(u_{\mathrm{F}}\right)=2 u_{\mathrm{F}}-10\right)$.

The philosophy in most cases is first to get an impression of what the leader can achieve and subsequently try to find a strategy to really achieve this goal. If one does not have any clue as to what the leader can achieve (in terms of maximal costs), hardly anything is known.

\section{Bi-level optimal toll design problem}

Consider a connected road network $\mathcal{G}=(\mathcal{N}, \mathcal{L})$, with a node set $\mathcal{N}=\{1, \ldots, n\}$ $(n \in \mathbb{N})$ and a set $\mathcal{L}=\left\{l_{1}, \ldots, l_{|\mathcal{L}|}\right\}(|\mathcal{L}| \in \mathbb{N})$ of directed links. Let $\mathcal{O D} \subset \mathcal{N} \times \mathcal{N}$ be a set of origin-destination pairs of the network. We denote the nonempty set of simple routes from the origin $o$ to the destination $d$ by $\mathcal{P}^{(o, d)}$ and the set of all simple routes of the network by $\mathcal{P}$. There is a fixed number of travelers traveling between each $(o, d)$-pair: $D^{(o, d)}$. Let us denote the number of travelers using the link $l_{j} \in \mathcal{L}$ (link flow) by $q_{l_{j}}$ and the number of travelers using the route $r_{i} \in \mathcal{P}$ (route flow) by $f_{r_{i}}$. Feasibility with respect to the number of travelers requires the route flows $\left[f_{r_{i}}\right]_{r_{i} \in \mathcal{P}^{(o, d)}}$ satisfy the conditions $[5,6]$ :

$$
\begin{gathered}
\sum_{r_{i} \in \mathcal{P}^{(o, d)}} f_{r_{i}}=D^{(o, d)}, \quad(o, d) \in \mathcal{O D} \\
f_{r_{i}} \geq 0, \quad r_{i} \in \mathcal{P}^{(o, d)}, \quad(o, d) \in \mathcal{O D} .
\end{gathered}
$$


We let $\left[\delta_{r_{i}, l_{j}}\right]_{r_{i} \in \mathcal{P}^{(o, d)}, l_{j} \in \mathcal{L},(o, d) \in \mathcal{O D}}$ be a link-route incidence matrix for $G$ with

$$
\delta_{r_{i}, l_{j}}=\left\{\begin{array}{c}
1 \quad \begin{array}{c}
\text { if route } r_{i} \in \mathcal{P}^{(o, d)} \\
\text { contains link } l_{j} \in \mathcal{L} \\
0 \quad \text { otherwise }
\end{array}
\end{array}\right.
$$

Then the link flows $q_{l_{j}}$ are defined by the route flows $f_{r_{j}}$ through

$$
q_{l_{j}}=\sum_{(o, d) \in \mathcal{O} \mathcal{D}} \sum_{r_{i} \in \mathcal{P}^{(o, d)}} \delta_{r_{i}, l_{j}} f_{r_{i}}, \quad l_{j} \in \mathcal{L} .
$$

With each link $l_{j} \in \mathcal{L}$ is associated the link cost defined as $c_{l_{j}}=\alpha \tau_{l_{j}}+\theta_{l_{j}}$, where $\alpha>0, \tau_{l_{j}}=\beta_{j} q_{l_{j}}+\delta_{j}\left(\beta_{j}, \delta_{j} \in \mathbb{R}_{+}\right)$is the link travel time on the link $l_{j}, \alpha$ is the travelers' value of time, and $\theta_{l_{j}}$ is the toll paid for usage of link $l_{j}$. The route $\operatorname{costs} c_{r_{i}},\left(r_{i} \in \mathcal{P}\right)$ are defined by the link costs $c_{l_{j}}\left(l_{j} \in \mathcal{L}\right)$ through $c_{r_{i}}=\sum_{l_{j} \in \mathcal{L}} \delta_{r_{i}, l_{j}} c_{l_{j}}$. We consider the deterministic traffic equilibrium assignment model ([6-8]) which is based on the assumption that all road users have complete information about the current traffic conditions, and they choose among routes available the cheapest one. In equilibrium state the travel costs on routes between one $(o, d)$-pair that are used are equal, i.e., they are all cheapest with respect to the current traffic flow.

Definition 3.1 (Deterministic User Equilibrium (DUE)) The deterministic (Wardrop) user equilibrium situation is described as follows:

$$
\begin{aligned}
f_{r_{i}}\left(c_{r_{i}}-\pi^{(o, d)}\right) & =0, \quad r_{i} \in \mathcal{P}^{(o, d)} \\
c_{r_{i}}-\pi^{(o, d)} \geq 0, & r_{i} \in \mathcal{P}^{(o, d)},
\end{aligned}
$$

where $\pi^{(o, d)}$ takes the role of the minimal travel cost of routes from $\mathcal{P}^{(o, d)}$.

Since travelers use the cheapest routes, they do not generally fulfil society's goal of efficient road usage, which may, for example, be a minimal total travel time. In order to fulfil this goal, during the last several years much attention has been paid to road pricing systems [3,9]. In such systems, each vehicle pays a prespecified toll for traversing certain links. By appropriate tolling the travelers can be forced to help the authority to achieve its goal. We consider two possible goals of the road authority: to minimize the total travel time or to maximize the total toll revenue.

\section{Case studies}

\subsection{Scenario 1}

Let us consider the one origin-destination network depicted in Figure 1 consisting of $|\mathcal{L}| \geq 2$ directed links.

We assume a one leader-more followers inverse Stackelberg game where the road authority as the leader sets link tolls as follows: The link $l_{|\mathcal{L}|}$ is untolled and 


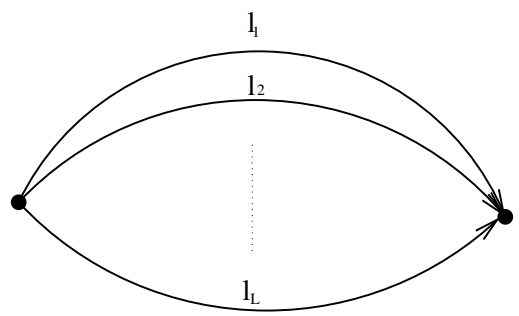

Figure 1: One origin-destination pair network.

each other link is tolled with a toll defined as a positive multiple of the number of travelers on the same link, i.e., $\theta_{l_{j}}=k_{j} q_{j}$ with $k_{j} \in \mathbb{R}_{+}$. Travelers as followers are driven by DUE defined in Definition 3.1. The cost for the traveler on the link $l_{j}$ is defined as $c_{l_{j}}=\alpha \tau_{l_{j}}+\theta_{l_{j}}=\alpha \beta_{j} q_{l_{j}}+\alpha \delta_{j}+k_{j} q_{l_{j}}$. We assume a positive number $D=D^{(o, d)}>0$. From DUE we derive that equations

$$
\begin{aligned}
q_{l_{i}} & =\frac{q_{l_{i+1}}\left(\alpha \beta_{i+1}+k_{i+1}\right)+\alpha\left(\delta_{i+1}-\delta_{i}\right)}{\alpha \beta_{i}+k_{i}}, \quad i \in\{1, \ldots|\mathcal{L}|-1\} \\
\sum_{j=1}^{|L|} q_{l_{j}} & =D
\end{aligned}
$$

have to be fulfilled. Because (5) forms a system of $|\mathcal{L}|$ linear equations of $|\mathcal{L}|$ unknowns, with given $D, l_{i}, \beta_{i}, \delta_{i}>0(i \in\{0, \ldots,|\mathcal{L}|\})$ a unique set $\left\{q_{l_{1}}, \ldots, q_{l_{|\mathcal{L}|}}\right\}$ solving (5) always exists provided the link flows are positive.

Now we focus on the optimal strategy for the road authority. With positive $D$, $\alpha, \beta_{i}, \delta_{i}(i \in\{1, \ldots,|\mathcal{L}|\})$ and $|\mathcal{L}|$ set to 2,3 , and 4 , respectively, we consider the same game as before with the objective functions of the road authority defined as both total travel time and total toll revenue. Trivially both total toll revenue and total travel time objective functions are smooth and twice differentiable with respect to all $k_{i}>0, i \in\{1, \ldots|\mathcal{L}|-1\}$. We find critical points of these functions and discuss conditions under which the critical points become local extrema.

\subsubsection{2 links with one origin-destination pair}

There is one critical point of the total travel time function and one critical point of the total toll revenue function, $k_{1}^{(*, 1)}=\frac{\left(-\beta_{2} \delta_{1}+\beta_{2} \delta_{2}+\delta_{2} \beta_{1}-\delta_{1} \beta_{1}\right) \alpha}{2 d \beta_{2}+\delta_{2}-\delta_{1}}, k_{1}^{(* *, 1)}=$ $\alpha \beta_{2}+\alpha \beta_{1} \in \mathbb{R}_{+}$, respectively.

The second derivative tests shows that if $\delta_{1}-\delta_{2} \neq 2 \beta_{2} D, \delta_{1}-\delta_{2} \neq \beta_{2} D$ the global minimum of the travel time function is reached at $k_{1}=k_{1}^{(*, 1)}$. Similarly if $\delta_{1}-\delta_{2} \neq 2 \beta_{2} D$ the global maximum of the total toll revenue function is reached at $k_{2}=k_{2}^{(* *, 1)}$. 


\subsubsection{3 links with one origin-destination pair}

There are two critical points of the total travel time function $\left(k_{1}^{(*, 1)}, k_{2}^{(*, 1)}\right)$, $\left(k_{1}^{(*, 2)}, k_{2}^{(*, 2)}\right)$ and only one critical point of the total toll revenue function $\left(k_{1}^{(* *, 1)}, k_{2}^{(* *, 1)}\right)$. Because these functions are rather large and not very informative, they are not given here. Using Hessian test we find out that there exist conditions on parameters $\alpha, \beta_{i}, \delta_{i}, D(i \in\{1,2,3\})$ under which $\left(k_{1}^{(*, 1)}, k_{2}^{(*, 1)}\right),\left(k_{1}^{(*, 2)}, k_{2}^{(*, 2)}\right)$ are both local extrema of the travel time functions. At $\left(k_{1}^{(* *, 1)}, k_{2}^{(* *, 1)}\right)$ the global maximum of the total toll revenue function is reached provided that certain conditions on input paramters are satisfied.

\subsubsection{4 links with one origin-destination pair}

The total travel time function has three critical points $\left(k_{1}^{(*, 1)}, k_{2}^{(*, 1)}, k_{3}^{(*, 1)}\right)$, $\left(k_{1}^{(*, 2)}, k_{2}^{(*, 2)}, k_{3}^{(*, 2)}\right),\left(k_{1}^{(*, 3)}, k_{2}^{(*, 3)}, k_{3}^{(*, 3)}\right)$ and there is one critical point of the total toll revenue function $\left(k_{1}^{(* *, 1)}, k_{2}^{(* *, 1)}, k_{3}^{(* *, 1)}\right)$. Similarly as in previous case these functions are rather large and very not-informative, hence they are not given here. Using Hessian test we find that there are conditions on $\alpha, \beta_{i}, \delta_{i}, D$, under which $\left(k_{1}^{(*, 1)}, k_{2}^{(*, 1)}, k_{3}^{(*, 1)}\right),\left(k_{1}^{(*, 2)}, k_{2}^{(*, 2)}, k_{3}^{(*, 2)}\right),\left(k_{1}^{(*, 3)}, k_{2}^{(*, 3)}, k_{3}^{(*, 3)}\right)$ are all local extrema of the travel time functions. At $\left(k_{1}^{(* *, 1)}, k_{2}^{(* *, 1)}, k_{3}^{(* *, 1)}\right)$ the global maximum of the total toll revenue function is reached provided that certain conditions on input parameters are satisfied.

\subsection{Scenario 2}

\subsubsection{One origin-destination pair}

Let us assume the network depicted in Figure 2 with $(o, d)=(1,6), \mathcal{L}=$ $\left\{l_{1}, l_{2}, l_{3}, l_{4}, l_{5}, l_{6}, l_{7}\right\}, \mathcal{P}=\mathcal{P}^{(1,6)}=\left\{r_{1}, r_{2}, r_{3}\right\}$ with $r_{1}=\left(l_{1}, l_{2}, l_{5}\right), r_{2}=$ $\left(l_{1}, l_{4}, l_{5}\right), r_{3}=\left(l_{3}, l_{6}, l_{7}\right)$. We consider 3 different games on this network with input parameters and tolling functions of the road authority defined in Table 1: P1, P2, P3.

The road authority has two possible objective functions: the total travel time function and the total toll revenue, travelers are driven by DUE.

In Figures 3 and 4 the graphs of objective functions of the road authority from PW1 are given (with $\theta_{l_{1}}=k q_{l_{1}}$ ) similarly the graphs of objective functions from PW2 (with $\theta_{l_{1}}=k q_{l_{1}}, \theta_{l_{4}}=l q_{l_{4}}$ ) are depicted in Figures 5 and 6 . There is a unique optimal solution for the road authority. Also the optimal strategy of the road authority for $\mathrm{P} 3$ is unique.

\subsubsection{Two origin-destination pairs}

We extend problem P1 with input parameters defined in Table 1 by adding one origin-destination pair $(2,6)$. The number of travelers traversing the roads from the set $\mathcal{P}^{(1,6)}=\left\{r_{1}, r_{2}, r_{3}\right\}$ with $r_{1}=\left(l_{1}, l_{2}, l_{5}\right), r_{2}=\left(l_{1}, l_{2}, l_{5}\right), r_{3}=$ $\left(l_{3}, l_{6}, l_{7}\right)$ is set to 12000 , the number of travelers using the roads from the set $\mathcal{P}^{(2,6)}=\left\{r_{4}, r_{5}\right\}$, with $r_{4}=\left(l_{2}, l_{5}\right), r_{5}=\left(l_{4}, l_{7}\right)$ is set to 4000 . Then travelers 
Table 1: Input data of the three different case studies using Scenario 2.

\begin{tabular}{|c|c|c|c|}
\hline & $\mathrm{P} 1$ & $\mathrm{P} 2$ & $\mathrm{P} 3$ \\
\hline$\left\{\beta_{1}, \beta_{2}, \beta_{3}\right\}$ & $\left\{\frac{1}{3000}, \frac{2}{3000}, \frac{2}{3000}\right\}$ & $\left\{\frac{1}{3000}, \frac{2}{3000}, \frac{2}{3000}\right\}$ & $\left\{\frac{1}{3000}, \frac{1}{3000}, \frac{1}{3000}\right\}$ \\
$\left\{\beta_{4}, \beta_{5}, \beta_{6}\right\}$ & $\left\{\frac{1}{12000}, \frac{2}{3000}, \frac{2}{3000}\right\}$ & $\left\{\frac{1}{12000}, \frac{2}{3000}, \frac{2}{3000}\right\}$ & $\left\{\frac{1}{3000}, \frac{1}{6000}, \frac{1}{3000}\right\}$ \\
$\beta_{7}$ & $\frac{2}{3000}$ & $\frac{2}{3000}$ & $\frac{1}{3000}$ \\
\hline$\left\{\delta_{1}, \delta_{2}, \delta_{3}, \delta_{4}\right\}$ & $\{1,2,2,2\}$ & $\{1,2,2,2\}$ & $\{1,1,2,2\}$ \\
$\left\{\delta_{5}, \delta_{6}, \delta_{7}\right\}$ & $\{2,2,2\}$ & $\{2,2,2\}$ & $\{1,1,1\}$ \\
\hline$\left\{\theta_{l_{1}}, \theta_{l_{4}}, \theta_{l_{5}}\right\}$ & $\left\{k \cdot q_{l_{1}}, 0,0\right\}$ & $\left\{k \cdot q_{l_{1}}, l \cdot q_{l_{4}}, 0\right\}$ & $\left\{0,0, k \cdot \frac{q_{l_{5}}}{f_{r_{3}}+f_{r_{1}}}\right\}$ \\
\hline
\end{tabular}

driven by DUE result in flows $f_{r_{1}}=\frac{24 \cdot 10^{3}+24 \cdot 10^{5} k+1875 k f r_{5}+14 f r_{5}}{14+1875 k}, f_{r_{2}}=$ $-\frac{1875 k f_{r_{5}}+24 \cdot 10^{5} k+14 f_{r_{5}}-128 \cdot 10^{3}}{14+1875 k}, f_{r_{3}}=4000 \frac{4+5625 k}{14+1875 k}$, and $f_{r_{4}}=4000-f_{r_{5}}$.

The link flow and the travel time on the tolled link $l_{1}$ are not functions of $f_{r_{5}}$ : $q_{l_{1}}=\frac{152000}{14+1875 k}$ and $\tau_{l_{1}}=\frac{194+5625 k}{3(14+1875 k)}$.

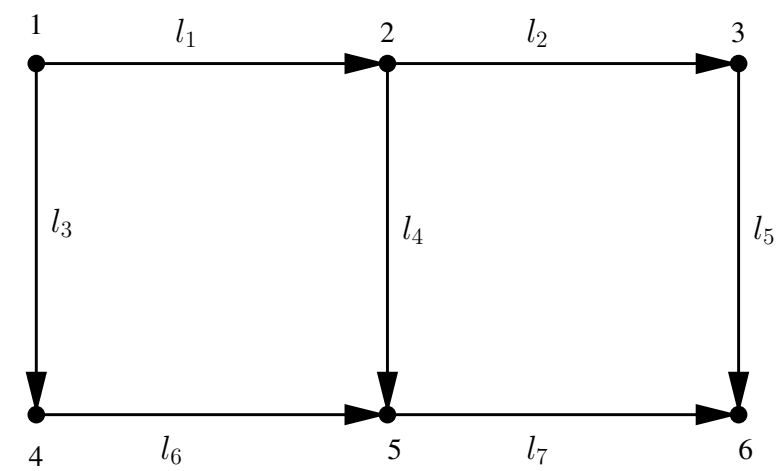

Figure 2: One origin-destination network.

The total travel time function is $F_{1}(k)=\frac{8000\left(17582+4205625 k+519328125 k^{2}\right)}{3(14+1875 k)^{2}}$ and there is a unique $k^{(*, 1)}=\arg \min _{k \geq 0} F_{1}(k)=\frac{66}{62275}$ such that $\theta_{l_{1}}^{*}=k^{(*, 1)} q_{l_{1}}$ is the optimal strategy for the road authority minimizing the total travel time. If the road authority chooses $\theta_{l_{1}}=k^{(*, 1)} q_{l_{1}}$, travelers will result in the route flows $f_{r_{1}}^{*}=\frac{217500}{131}+f_{r_{5}}^{*}, f_{r_{2}}^{*}=-f_{r_{5}}^{*}+\frac{1028000}{131}, f_{r_{3}}^{*}=\frac{326500}{131}, f_{r_{4}}^{*}=4000-f_{r_{5}}^{*}, f_{r_{5}}^{*} \in$ $[0,4000]$.

The total toll revenue function is $F_{2}(k)=\frac{23104 \cdot 10^{6} k}{(14+1875 k)^{2}}$ and there is a unique $k^{(* *, 1)}=\arg \max _{k \geq 0} F_{2}(k)=\frac{14}{1875}$ such that $\theta_{l_{1}}^{*}=k^{(* *, 1)} q_{l_{1}}$ is the optimal strategy for the road authority maximizing the total toll revenue. If the road authority chooses $\theta_{l_{1}}=k^{(* *, 1)} q_{l_{1}}$, travelers will result in the route flows $f_{r_{1}}^{*}=$ $\frac{10480}{7}+f_{r_{5}}^{*}, f_{r_{2}}^{*}=-f_{r_{5}}^{*}+\frac{27520}{7}, f_{r_{3}}^{*}=\frac{46000}{7}, f_{r_{4}}^{*}=4000-f_{r_{5}}^{*}, f_{r_{5}}^{*} \in\left[0, \frac{27520}{7}\right]$. 


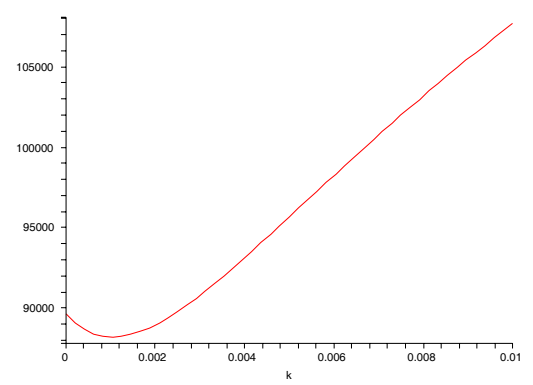

Figure 3: Total travel time - P1.

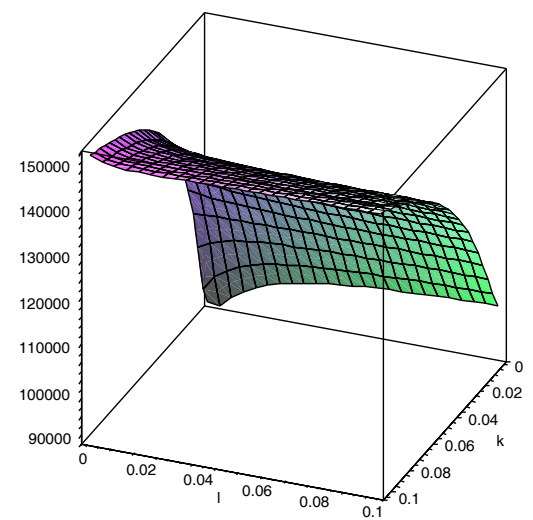

Figure 5: Total travel time - P2.

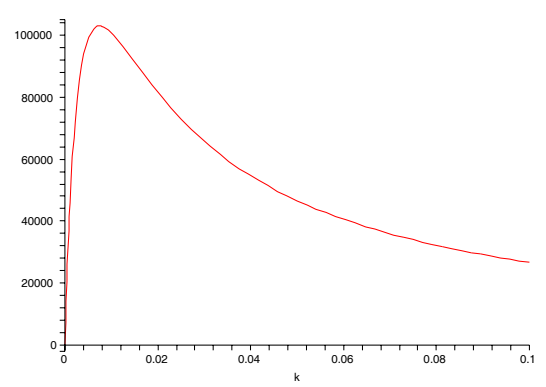

Figure 4: Total toll revenue - P1.

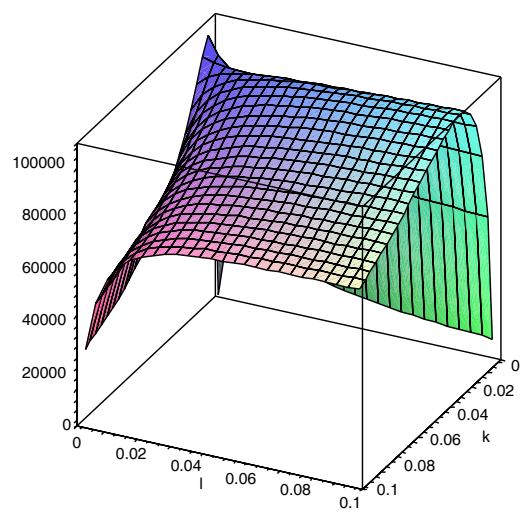

Figure 6: Total toll revenue - P2.

There is a unique optimal strategy of the road authority and infinity many strategies of the travelers.

\section{Conclusions}

We introduced inverse Stackelberg games and their application in the bi-level optimal toll design problems. Using case studies with toll to be often defined as a linear function proportional to the link flows we showed that the solution does not need to be unique for both road authority and travelers.

The problem is generally not-convex, and hence classical convex optimization methods cannot be used.

In the future work we will consider bi-level optimal toll design problems with stochastic user equilibrium traffic assignment as well as extension to larger networks. 


\section{References}

[1] Basar, T. \& Olsder, G., Dynamic Noncooperative Game Theory. SIAM: Philadelphia, 1999.

[2] Shen, H. \& Basar, T., Incentive-based pricing for network games with complete and incomplete information. Annals of Dynamic Games, 8, 2006.

[3] Joksimovič, D., Bliemer, M. \& Bovy, P., Optimal toll design problem in dynamic trafic networks - with joint route and departure time choice. Transportation Research Records, 2004.

[4] Olsder, G., Phenomena in inverse Stackelberg problems. Regelungstheorie 11, Mathematisches Forschungsinstitut Oberwolfach, Germany, pp. 603-605, 2005.

[5] Chen, K.H., Dynamic Travel Choice Models: A Variational Inequality Approach. Springer: Oxford, UK, 1999.

[6] Patriksson, M., The Traffic Assignment Problem: Models and Methods. VSP: The Netherlands, 1994.

[7] Patriksson, M., Nonlinear programming and variational inequality problems: A unified approach. Kluwer.: The Netherlands, 1999.

[8] Lolito, P., Quadrat, J.P. \& Mancinelli, E., Traffic assignment \& Gibbs-Maslov semirings. Contemporary Mathematics, 377, pp. 209-219, 2005.

[9] Joksimovič, D., Bliemer, M., Bovy, P. \& Verwater-Lukszo, Z., Dynamic road pricing for optimizing network performance with heterogenous users. Proceedings of IEEE International Conference in Networking, Sensing and Control, Tucson, Arizona, USA, pp. 407-412, 2005. 